\title{
Kondo effect and superconductivity in niobium with iron impurities
}

\author{
Hansong Zeng ${ }^{1}$, Dan Zhou ${ }^{1}$, Guoqing Liang ${ }^{1}$, Rujun Tang ${ }^{1 凶}$, Zhi H. Hang ${ }^{1}$, Zhiwei Hu ${ }^{2}$, \\ Zixi Pei ${ }^{2} \&$ X. S. Ling ${ }^{3 凶}$
}

Kondo effect is an interesting phenomenon in quantum many-body physics. Niobium ( $\mathrm{Nb}$ ) is a conventional superconductor important for many superconducting device applications. It was long thought that the Kondo effect cannot be observed in $\mathrm{Nb}$ because the magnetic moment of a magnetic impurity, e.g. iron ( $\mathrm{Fe}$ ), would have been quenched in $\mathrm{Nb}$. Here we report an observation of the Kondo effect in a $\mathrm{Nb}$ thin film structure. We found that by co-annealing Nb films with $\mathrm{Fe}$ in Argon gas at above $400^{\circ} \mathrm{C}$ for an hour, one can induce a Kondo effect in $\mathrm{Nb}$. The Kondo effect is more pronounced at higher annealing temperature. The temperature dependence of the resistance suggests existence of remnant superconductivity at low temperatures even though the system never becomes superconducting. We find that the Hamann theory for the Kondo resistivity gives a satisfactory fitting to the result. The Hamann analysis gives a Kondo temperature for this $\mathrm{Nb}-\mathrm{Fe}$ system at $\sim 16 \mathrm{~K}$, well above the superconducting transition onset temperature $9 \mathrm{~K}$ of the starting $\mathrm{Nb}$ film, suggesting that the screening of the impurity spins is effective to allow Cooper pairs to form at low temperatures. We suggest that the mechanism by which the Fe impurities retain partially their magnetic moment is that they are located at the grain boundaries, not fully dissolved into the bcc lattice of $\mathrm{Nb}$.

Effects of impurities on superconductivity have been of longstanding interest in quantum condensed-matter physics ${ }^{1}$. According to Anderson's theorem ${ }^{2}$, nonmagnetic impurities should have little effect on the superconducting transition temperature $T_{\mathrm{c}}$. Magnetic impurities cause spin-flip scattering of the conduction electrons thus will have a large effect on the Cooper pairing in a superconductor, i.e. suppressing $T_{\mathfrak{c}}$, as indeed observed ${ }^{3}$. However, a striking exception ${ }^{4}$ was that dilute amount of Fe dissolved in $\mathrm{Nb}$ seems to have little effect on the superconducting transition temperature, and magnetic susceptibility measurement showed ${ }^{3,4}$ that the magnetic moment of $\mathrm{Fe}$ was absent in the host metal Nb. Anderson explained ${ }^{5}$ that whether a magnetic impurity ion can retain its magnetic moment depends on the density of states at the Fermi energy $E_{F}$ of the host metal. The Anderson model explains nicely why Fe in molybdenum is magnetic and can produce a Kondo effect, while Fe in $\mathrm{Nb}$ cannot, since $\mathrm{Nb}$ has a larger density of state at $E_{F}$ than molybdenum ${ }^{6}$. Thus what we are reporting here came first as a surprise that when we introduced a very dilute amount of $\mathrm{Fe}$ into a $\mathrm{Nb}$ thin film, we observed a drastic reduction in $T_{\mathrm{c}}$ and a well developed Kondo effect. Details of our observation will be described below.

The Kondo effect refers to an anomalous minimum in the temperature dependence of resistivity in a metal first observed in gold wires ${ }^{7}$ and later Kondo ${ }^{8}$ discovered that the anomalous increase of resistivity with decreasing temperature can arise from a strong (divergent) spin-flip scattering between the magnetic impurities and the conducting electrons of the host metal when their exchange interaction is antiferromagnetic. Later improved theories ${ }^{9-11}$ showed that with decreasing temperature, the spin-flip scattering on the conduction electrons due to the localized spin on magnetic impurities do indeed increase with decreasing temperature, however, there is no divergence at $T=0$. Instead, the localized spin and the conduction electron spins form a virtual many-body bound state below a characteristic temperature $T_{\mathrm{K}}$. At $T=0$ the localized spin is completely screened. There is no divergence in resistivity as $T$ approaches absolute zero. Further theoretical improvements were also provided to account for the orbital angular momentum of the impurity atoms ${ }^{12}$.

Early experimental studies of Kondo effect were on metals ${ }^{7,13-17}$, recently there have been reports of Kondo effect in materials like oxide films and carbon-based materials ${ }^{18-21}$, and most recently in transition metal dichalcogenides ${ }^{22}$. Various methods have been used to introduce magnetic impurities into the host material, such as chemical vapor transport method, melt growth method and paramagnetic gating, etc. ${ }^{23-27}$.

Superconductivity emerges from another nontrivial bound state in a many-body quantum system: Cooper's pairing ${ }^{28}$ of two electrons with opposite spins and momenta on the Fermi surface mediated by the

${ }^{1}$ Institute for Advanced Study, School of Physical Science and Technology, Soochow University, Suzhou 215006, People's Republic of China. 'Institute of Physics, Chinese Academy of Science, Beijing 100190, People's Republic of China. ${ }^{3}$ Department of Physics, Brown University, Providence, RI 02912, USA. ${ }^{\square}$ email: tangrj@suda.edu.cn; xinsheng_ling@brown.edu 
electron-phonon interactions. The formation of a phase coherent ground state of many Cooper pairs results in resistance vanishing when the temperature is lowered to below $T_{\mathrm{c}}{ }^{29}$. When magnetic impurities are incorporated into the system, the interplay between these two competing ground states has been a subject of great interest ${ }^{1,30-35}$. In superconducting alloys, Kondo effect can change its superconducting properties greatly ${ }^{36-38}$. The competition between superconductivity and Kondo effect has been of great interests ${ }^{39-42}$. In recent years, the Kondo effect has also been studied in many new superconductors materials ${ }^{31-35}$, in $2 \mathrm{D}$ topological superconductors and 3D topological insulators ${ }^{43,44}$. Similar resisivity minimum was also observed in high- $T_{\mathrm{c}}$ superconductors ${ }^{45}$.

$\mathrm{Nb}$ is an important superconducting material for its stability and for having the highest superconducting transition temperature $(9.2 \mathrm{~K})$ among the elemental metals. Superconducting properties of Nb have been investigated intensely over the years ${ }^{46-51}$ and in theories ${ }^{52,53}$. Thin films of $\mathrm{Nb}$ are of great interest for model systems ${ }^{54}$ and devices ${ }^{55}$. $\mathrm{NbSe}_{2}$ is a close relative of $\mathrm{Nb}$, a recent experiment ${ }^{56}$ revealed that superconductivity and magnetism can coexist in $\mathrm{NbSe}_{2}$. Thus it is of great interest to explore if one can create Kondo effect in $\mathrm{Nb}$ as well in spite of early studies ${ }^{3,4,6}$ that suggested the quenching of Fe magnetic moment in $\mathrm{Nb}$. Here we report transport measurements of sputtered $\mathrm{Nb}$ thin films containing low density of Fe impurities that clearly demonstrate the existence of the Kondo effect in a $\mathrm{Nb}$ thin film structure.

\section{Results and discussion}

The effects of co-annealing with Fe. Figure 1 shows the main findings of this work. Figure la shows the temperature dependence of the resistivity of an as-sputtered sample in zero field and at $5 \mathrm{~T}$. The superconducting transition temperature $T_{\mathrm{c}}$ is defined as the temperature at which the sample resistivity has dropped to $1 / 2$ of its normal state value (at $10 \mathrm{~K}$ ). The onset temperature of superconductivity $T_{c}^{*}$ is defined as the data point where the resistivity shows a clear drop. The as-sputtered sample shows $T_{\mathrm{c}}=8.875 \mathrm{~K}$ in zero magnetic field. At $5 \mathrm{~T}$, the $\rho-T$ is a smooth monotonic function of temperature down to $2.0 \mathrm{~K}$.

Figure $1 \mathrm{~b}$ shows the temperature dependence of the resistivity of the sample annealed at $300{ }^{\circ} \mathrm{C}$, also in zero field and at $5 \mathrm{~T}$. Immediately one notices the normal state resistivity is increased and the superconducting transition $T_{\mathrm{C}}$ is reduced. Yet, the $\rho-T$ at $5 \mathrm{~T}$ behaves very similar to that of the as-sputtered sample in Fig. $2 \mathrm{a}$. The $\rho-T$ curve at $5 \mathrm{~T}$ is also a monotonic function of temperature.

Figure $1 \mathrm{c}-\mathrm{e}$ show the $\rho-T$ curves (at $0 \mathrm{~T}$ and $5 \mathrm{~T}$ ) for the samples annealed at $400{ }^{\circ} \mathrm{C}, 500{ }^{\circ} \mathrm{C}$, and $600{ }^{\circ} \mathrm{C}$, respectively. Two distinct features are immediately obvious to the eyes: there is a minimum in the $\rho-T$ in zero field and at $5 \mathrm{~T}$, and the sharp zero-field superconducting transition is replaced by a gradual decrease in resistivity. At $5 \mathrm{~T}$, the $\rho-T$ curves show a minimum around $20 \mathrm{~K}$. Between 5 and $20 \mathrm{~K}$, the $\rho-T$ curves show the characteristic logarithmic dependence o temperature often found in Kondo effect systems ${ }^{7,8}$. In Fig. 1f, the data in Fig. 1e are re-plotted in a semi-log plot, with $x$-axis being logarithmic. The regime of $-\ln T$ is marked by a dashed straightline, indicating the logarithmic behavior characteristic of the Kondo effect.

Understanding the "normal" behavior. In Fig. 1a,b, the temperature dependence of the resistivity in a magnetic field of $5 \mathrm{~T}$, when the superconductivity in the system is suppressed, behave as expected for "pure" $\mathrm{Nb}$ films. With decreasing temperature, the resistivity decreases monotonically towards a constant value. We made an attempt to fit these two data sets to the normal metal resistivity formula ${ }^{57} \rho(T)=\rho_{0}+a T^{2}+b T^{5}$ that contains the residual resistivity (constant $\rho_{0}$ ), the Fermi liquid contribution (electron-electron scattering, $\sim T^{2}$ ), and the electron-phonon interaction $\left(\sim T^{5}\right)$. The fitting (not shown) is reasonably well considering the fact that it did not consider the effects of grain boundary scattering ${ }^{58}$ and surface scattering ${ }^{59}$. In the early study of the normal state resistivity of $\mathrm{Nb}^{48}$, it was argued that there should be a $T^{3}$ term in the $\rho-T$ due to the inter-band scattering ( $\mathrm{Nb}$ conduction band consists of a mixture of $s-d$ band $s^{53}$ ), in addition to the three terms discussed above. We found that by adding a $T^{3}$ term can indeed improve on the data fitting to almost perfection, however, the fitting parameters for the coefficients for the Fermi liquid $T^{2}$ and the electron-phonon $T^{5}$ terms become negative, i.e. nonphysical. Thus we believe the standard three terms are adequate description of the normal state resistivity in our as-sputtered and $300{ }^{\circ} \mathrm{C}$ annealed $\mathrm{Nb}$ films.

Figure $1 \mathrm{~b}$ deserves some special attention. After co-annealing at $300{ }^{\circ} \mathrm{C}$, the $T_{\mathrm{c}}$ of the $\mathrm{Nb}$ film is reduced slightly, by $1.4 \mathrm{~K}$, yet there is no Kondo effect even at $5 \mathrm{~T}$ magnetic field when the superconductivity is fully suppressed. We believe this $T_{\mathrm{c}}$ reduction is the formation of $\mathrm{NbO}$ due to the substrate $\mathrm{SiO}_{2}$, as also found previously ${ }^{60}$. $\mathrm{NbO}$ is a metal which becomes superconducting at $1.2 \mathrm{~K}^{61}$. Thus it is likely the layer of $\mathrm{Nb}$ that is in intimate contact with $\mathrm{SiO}_{2}$ layer is converted to $\mathrm{NbO}$ which then reduces the $T_{\mathrm{c}}$ of the top layer of $\mathrm{Nb}$ by the proximity effect ${ }^{62}$. It should be noted that $\mathrm{NbO}$ is itself an interesting quantum material ${ }^{63,64}$.

Ruling out localization. In disordered metals, in addition to the Kondo effect, there is another mechanism that can give rise to an anomalous increase in resistance as a function of decreasing temperature, namely the weak localization (for a review, $\mathrm{se}^{65}$ ). At very low temperatures, the quantum interference between the elastic scattering paths in a disordered metal can cause additional resistance. This resistance decreases with increasing temperature, before the eventual rise again in resistance due to the electron-phonon scattering. This weak localization effect can also lead to a resistance minimum without any magnetic impurities. We believe this is not what happens in our samples. For weak localization, adding magnetic field will suppress the quantum interference effects, resulting in a negative magneto-resistance ${ }^{66}$. Here, as shown in Fig. 2, the magneto-resistance below $30 \mathrm{~K}$ is positive for all samples.

As shown in Fig. 2, the $R$ vs. $T$ curves at different magnetic fields are shown, for the samples annealed at (a) $400{ }^{\circ} \mathrm{C}$; (b) $500{ }^{\circ} \mathrm{C}$; (c) $600{ }^{\circ} \mathrm{C}$. They all clearly show a resistance minimum around a $T_{\min } \sim 10-20 \mathrm{~K}$. We also tried annealing in shorter time, $30 \mathrm{~min}$., the effects are equivalent to that at lower temperatures for a longer time. For samples annealed at $400{ }^{\circ} \mathrm{C}, 500{ }^{\circ} \mathrm{C}$ and $600{ }^{\circ} \mathrm{C}$, the resistance minima $T_{\text {min }}$ appear at $\sim 14 \mathrm{~K}, 16 \mathrm{~K}$ and 20 

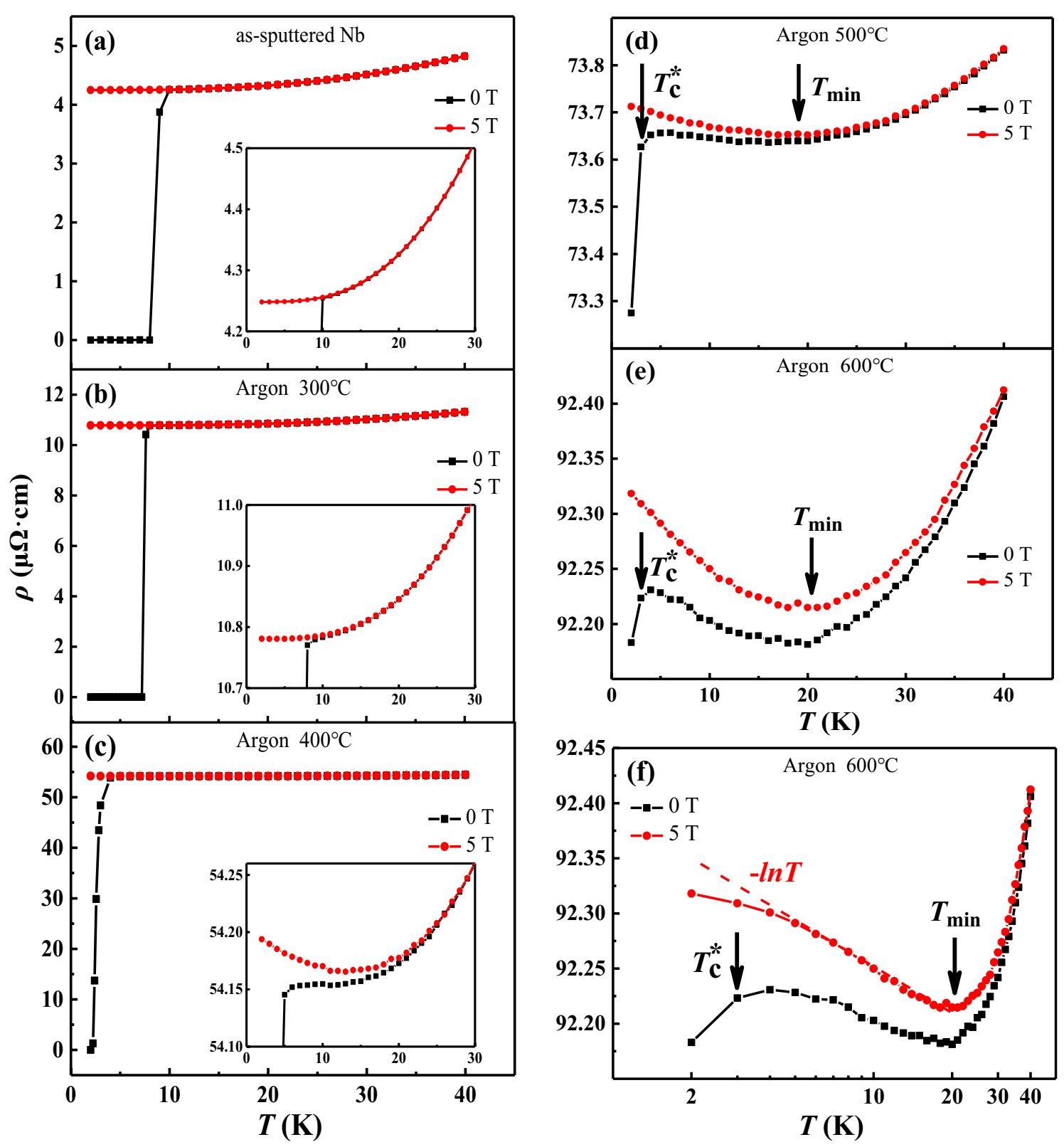

Figure 1. The effects of co-annealing with Fe in argon gas: (a-e) are the temperature dependence of resistivity $\rho$ vs. $T$ at $0 \mathrm{~T}$ and $5 \mathrm{~T}$ for as-sputtered $\mathrm{Nb}$ film, annealed at $300{ }^{\circ} \mathrm{C}, 400{ }^{\circ} \mathrm{C}, 500{ }^{\circ} \mathrm{C}$ and $600^{\circ} \mathrm{C}$, respectively. The annealing was done in flowing argon gas (pressure $50 \mathrm{~Pa}$ ), for $60 \mathrm{~min}$; (f) The data in (e) are re-plotted in a semi$\log$ plot, with the $x$-axis being in logarithmic scale. The regime of $-\ln T$ is marked by a dashed straight line. The onset of superconducting transition $T_{\mathrm{c}}^{*}$ is defined as the first drop of $\rho$.

$\mathrm{K}$, respectively. In Kondo's model ${ }^{8}$, with decreasing temperature, the resistance minimum is where the scattering from the magnetic impurities start to dominate in the electron transport (we should point out that the charge carriers are hole-like in $\mathrm{Nb}$ due to the anisotropic shape of its band structure ${ }^{67}$ ).

Below $T_{\min }$, in zero magnetic field, the resistance increases with decreasing temperature, then reaches a maximum and starts to decrease again. For the $400{ }^{\circ} \mathrm{C}$-annealed sample, the resistance drops to zero near $2 \mathrm{~K}$, while for $500^{\circ} \mathrm{C}$ and $600^{\circ} \mathrm{C}$-annealed samples, the resistance remains at a large value down to $2 \mathrm{~K}$. Nevertheless, we attribute the low- $T$ decrease in resistance to the onset of superconductivity in the sample, or more precisely small regions of the sample becoming superconducting, even though the system as a whole does not have a percolating path of superconducting regions. This picture is confirmed when we apply magnetic field. As shown in Fig. $2 \mathrm{a}-\mathrm{c}$, at field larger than $3 \mathrm{~T}$, the resistance increases with decreasing temperature to approach a saturation. The superconductivity is completely destroyed.

Choosing theoretical framework for data analysis: the Hamann formula. To analyze the $R-T$ curves in Fig. 2, we need a theoretical framework for the data analysis. In the original Kondo model ${ }^{8}$, the resistivity has a logarithmic dependence on temperature, or $-\ln \left(T / T_{\mathrm{K}}\right)$, where $T_{\mathrm{K}}$ is the Kondo temperature. In Fig. 2 


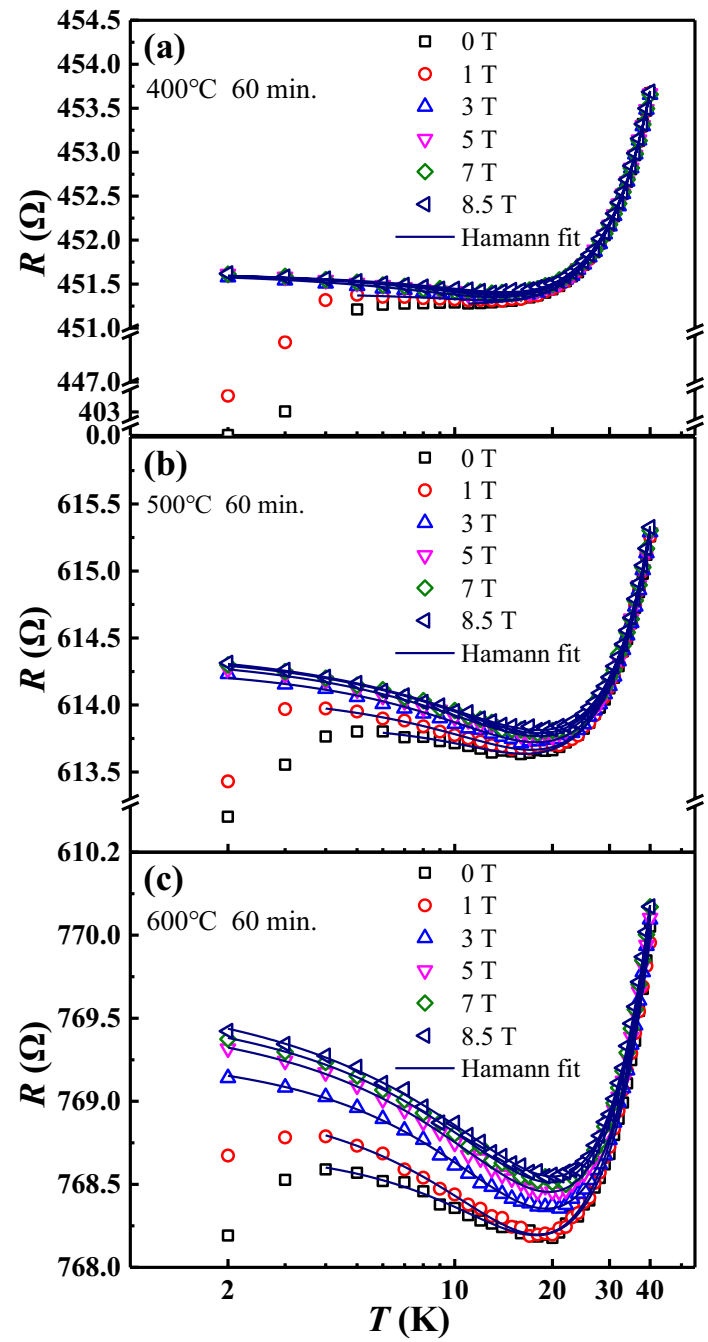

Figure 2. The effects of magnetic field on the $R-T$ for $\mathrm{Nb}$ samples co-annealed at (a) $400{ }^{\circ} \mathrm{C},(\mathbf{b}) 500{ }^{\circ} \mathrm{C}$, and (c) $600{ }^{\circ} \mathrm{C}$. The annealing time is $60 \mathrm{~min}$. for all. The magnetic field ranges from 0 to $8.5 \mathrm{~T}$. The roomtemperature resistance $R$ for the three samples: (a) $532.9 \Omega$, (b) $688.1 \Omega$ and (c) $861.7 \Omega$, respectively. The solid lines are the fitting curves for the Hamann theory. Note that the $x$-axes are in logarithmic scale.

we plot the resistance vs. $\log (T)$. There is indeed a region below $T_{\min }$ that the $R(T)$ curves have a logarithmic dependence on temperature. Upon further decrease in temperature, however, the resistance levels off. This feature was not explained by Kondo ${ }^{8}$. It was later understood that the logarithmic divergence as $T$ approaches absolute zero in Kondo's model is avoided by a screening effect of the impurity spin by a cloud of antiparallel spins from the conduction electrons, due to their antiferromagnetic exchange interactions ${ }^{9,11,68}$. This occurs below $T_{\mathrm{K}}$. The screening of the impurity spin by the Kondo cloud ${ }^{69}$ leads to a levelling off in the scattering rate (hence resistivity) as $T$ approaches absolute zero.

For analyzing experimental results on temperature-dependent resistivity, however, researchers need an analytical formula. The resistivity formula given by Hamann ${ }^{11}$ was the closest in describing experimental results ${ }^{15}$. In early experiments people adopted an empirical approach to mimic theoretical results of complex forms s $^{13,17}$. Recently, using empirical approach for the numerical renormalization group (NRG) result has gained wide popularity ${ }^{70-74}$. We also attempted to use this empirical NRG approach to fit our data in Fig. 2. However, we found that there is an underlying inconsistency in the analysis: the extracted $T_{\mathrm{K}}$ is much larger than $T_{\min }$, where the resistance clearly deviates from the normal metallic behavior (Drude + Fermi liquid + electron-phonon scattering).

Thus we decide to follow a recent study of the Kondo effect in $\mathrm{VSe}_{2}{ }^{75}$ and adopt the resistivity formula derived by Hamann ${ }^{11}$ using the theoretical approach proposed by Nagaoka ${ }^{10}$,

$$
R(T)=R_{0}+q T^{2}+p T^{5}+R_{1}\left(1-\frac{\ln \left(\frac{T}{T_{\mathrm{K}}}\right)}{\left[\ln ^{2}\left(\frac{T}{T_{\mathrm{K}}}\right)+S(S+1) \pi^{2}\right]^{1 / 2}}\right),
$$




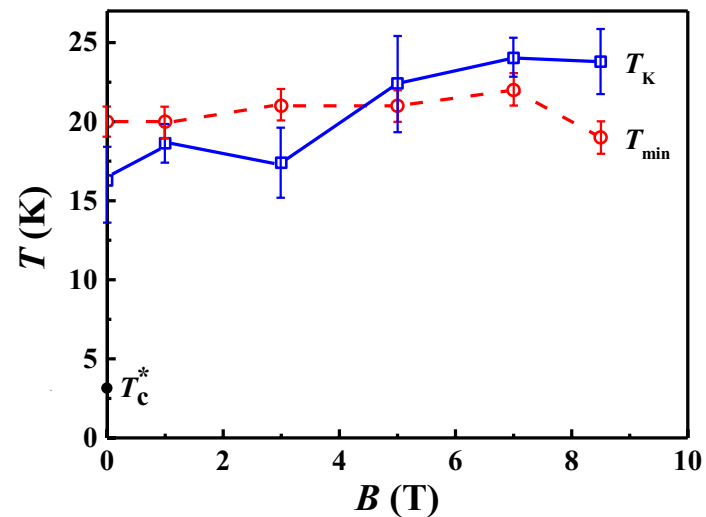

Figure 3. Characteristic temperatures vs. magnetic field B. $T_{\mathrm{K}}$ is the extracted Kondo temperature from Fig. 2 using the Hamann theory. $T_{\min }$ is the temperature where the resistance vs. temperature curve has a minimum, $T_{c}^{*}$ is the zero-field onset temperature for superconductivity. All are deduced from Fig. 2c.

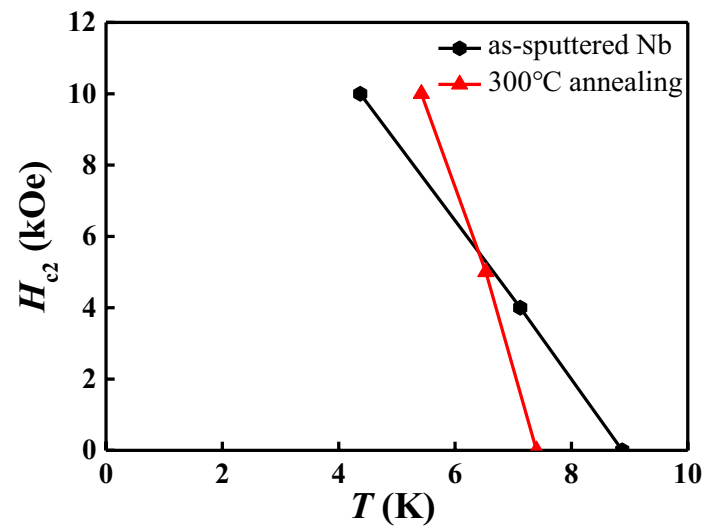

Figure 4. The upper critical fields $H_{c 2}(T)$ lines for the as-sputtered $\mathrm{Nb}$ and the $300{ }^{\circ} \mathrm{C}$ co-annealed $\mathrm{Nb}$ films. The superconducting transition temperature $T_{\mathrm{c}}$ is defined at $\rho\left(T_{\mathrm{c}}\right)=\rho_{\mathrm{n}}(10 \mathrm{~K}) / 2$. The critical temperature $T_{\mathrm{c}}(0)$ of the as-sputtered $\mathrm{Nb}$ film is $8.875 \mathrm{~K}$, and the superconducting transition width is about $52 \mathrm{mK}\left(10-90 \% R_{\mathrm{n}}\right.$ criterion).

where $R_{0}$ (Drude), $q$ (Fermi liquid), $p$ (electron-phonon), $R_{1}$ (Hamann's unitarity limit), $T_{\mathrm{K}}$ (Kondo) are fitting parameters.

The fitting curves using Eq. 1 are shown in Fig. 2 overlaying on top of the experimental data points. The agreement is striking. We found that if we set the impurity spin $S=1 / 2$ or larger, the fitting routine cannot converge. This was known to the field ${ }^{16,22}$ for quite sometime, and perhaps the main motivation for the empirical approaches ${ }^{13,17,22,70}$. Thus we also kept $S$ as a fitting parameter. We find that the extracted $S=0.1-0.17$, the extracted Kondo temperature $T_{\mathrm{K}}=15-24 \mathrm{~K}$, for magnetic field $=0-8.5 \mathrm{~T}$.

Kondo temperature $T_{\mathrm{K}}$ and $T_{\min }$. In Fig. 3, we compare the Kondo temperature with the resistance minimum temperature $T_{\min }$. The extracted Kondo temperatures $T_{\mathrm{K}}$ are consistent with the physical picture of Kondo ${ }^{8}$ that the spin-flip scattering starts to dominate at temperatures around the resistance minimum. In comparison, the Kondo temperatures $T_{\mathrm{K}}$ extracted using the empirical NRG approach are much larger than $T_{\min }$. In comparison, the remnant superconductivity sets in at $T_{\mathrm{c}}^{*}=3 \mathrm{~K}$. This is consistent with the expectations that the localized spin on Fe is sufficiently screened that the electron-phonon interaction is strong enough to create localized, non-coherent, superconducting regions.

Coherence length vs. mean free path. To gain some insights into the nature of the electronic states in the as-sputtered and $300{ }^{\circ} \mathrm{C}$ annealed $\mathrm{Nb}$ films, i.e. the "normal" films, we measured the $H_{\mathrm{c} 2}-T$ phase diagrams near $T_{\mathrm{c}}$ for these two samples, as shown in Fig. 4. The $H_{\mathrm{c} 2}(T)$ curves show linear temperature dependence near $T_{\mathrm{c}}$, in agreement with the Ginzburg-Landau (GL) theory ${ }^{62}$. From the slopes of the $H_{\mathrm{c} 2}(T)$ curves, using the GL theory we estimate $\xi$ to be about $12.9 \mathrm{~nm}$ for the as-sputtered sample, and $6.2 \mathrm{~nm}$ for the annealed at $300{ }^{\circ} \mathrm{C}$ sample. We also estimated the Drude mean free paths $l=\hbar k_{F} / n e^{2} \rho_{x x}$ using their $10 \mathrm{~K}$ resistivities, $l \sim 18.5 \mathrm{~nm}$ for the as-sputtered, and $7.2 \mathrm{~nm}$ for the annealed at $300{ }^{\circ} \mathrm{C}$ samples, respectively. These results suggest that the 


\begin{tabular}{|l|l|l|l|l|l|}
\hline Annealing temperature $\left({ }^{\circ} \mathbf{C}\right)$ & $\boldsymbol{T}_{\mathbf{c}}(\mathbf{K})$ & $\boldsymbol{T}_{\mathbf{c}}^{*}(\mathbf{K})$ & $\rho(\mu \boldsymbol{\Omega} \mathbf{c m})(\mathbf{1 0} \mathbf{K})$ & $\boldsymbol{l}(\mathbf{n m})(\mathbf{1 0} \mathbf{K})$ & $\xi(\mathbf{n m})$ \\
\hline 0 (as-sputtered $\mathrm{Nb})$ & 8.875 & 9 & 4.25 & 18.5 & 12.9 \\
\hline 300 & 7.4 & 7.6 & 10.8 & 7.2 & 6.2 \\
\hline 400 & 2.4 & 3 & 54.2 & 1.46 & - \\
\hline 500 & - & 3 & 73.6 & 1.07 & - \\
\hline 600 & - & 3 & 92.2 & 0.86 & - \\
\hline
\end{tabular}

Table 1. Summary of the parameters measured from the as-sputtered $\mathrm{Nb}$ film and the annealed samples.

coherence length is of the same order of magnitude as the mean free paths. Since $1 / \xi=1 / \xi_{0}+1 / l$, where $\xi_{0}$ is the intrinsic coherence length, $l$ is the mean free path, we conclude that the superconducting coherence length in our $\mathrm{Nb}$ films is determined by the electronic mean free paths. In Table 1, we summarize some of the key parameters of the five samples discussed in Fig. 1.

Why is the magnetic moment of Fe not quenched in Nb thin films? It is well-known that the magnetic moment of $\mathrm{Fe}$ impurities will be quenched when the Fe atoms are dissolved in $\mathrm{Nb}^{3,4,6}$. In those systems, mixing of $\mathrm{Fe}$ into $\mathrm{Nb}$ was done at $1200^{\circ} \mathrm{C}$ for a time period of a week. It is expected that Fe atoms are well incorporated into the bcc lattice of $\mathrm{Nb}$, even though their exact locations are unknown. Here, given the highest temperature we used, $600{ }^{\circ} \mathrm{C}$, the $\mathrm{Fe}$ atoms are expected to be adsorbed between the crystalline grains, i.e. not fully dissolved into the bcc lattice of the $\mathrm{Nb}$ crystalline grains. The fact that the extracted spin parameter $S \sim$ 0.10-0.17 is small is consistent with Anderson's theory ${ }^{5}$ on local magnetic moment formation in metals.

The sign of magneto-resistance. The sign of the magneto-resistance of the Kondo effect contribution should be negative since the magnetic field tends to destablize the virtual bound state between the localized moment and the conduction electrons. As shown in Fig. 2, the sign of magneto-resistance here is positive, the resistance increases with magnetic field. In the $\mathrm{Au}-\mathrm{Fe}$ system ${ }^{76}$, the raw data of magneto-resistance was also positive, only after subtracting out the non-Kondo part can one see the negative magneto-resistance. Unfortunately, such a subtraction procedure cannot be carried out for the $\mathrm{Nb}-\mathrm{Fe}$ system here due to the residual superconductivity in the system. As shown in Supplementary Fig. SI-2 (in Supplementary Information), we re-plot the data in Fig. $2 \mathrm{c}$ as a function of magnetic field for the $\mathrm{Nb}$ sample annealed at $600^{\circ} \mathrm{C}$. In Supplementary Fig. SI-2a, below $10 \mathrm{~K}$, there is a large positive magneto-resistance effect due to the destruction of the Cooper pairs with increasing field. Above $10 \mathrm{~K}$, shown in Supplementary Fig. SI-2b, the magneto-resistance effect is small, but still positive. This is the well known "classical" magneto-resistance of metals due to grain boundaries and Fermi surface anisotropy as discussed by Giordano ${ }^{76}$. However, due to the presence of the residual superconductivity in the $\mathrm{Nb}-\mathrm{Fe}$ system here, the subtraction procedure of Giordano ${ }^{76}$ cannot be applied. Thus we rely on the curve fitting using Hamann's theory ${ }^{11}$.

\section{Conclusions}

We report a resistance anomaly of sputtered $\mathrm{Nb}$ films after co-annealing with Fe in inert gas at different temperatures up to $600{ }^{\circ} \mathrm{C}$. We found that with an increase of annealing temperature from 300 to $600{ }^{\circ} \mathrm{C}$, the superconducting transition temperature $\left(T_{\mathrm{c}}\right)$ of $\mathrm{Nb}$ film changes sharply from $8.9 \mathrm{~K}$ to below $2 \mathrm{~K}$, even though there are hints of superconductivity remaining below $3 \mathrm{~K}$ for samples annealed at $600{ }^{\circ} \mathrm{C}$ for $60 \mathrm{~min}$. Moreover, for films annealed at above $400^{\circ} \mathrm{C}$, a $R-T$ resistance minimum is observed which persists under different magnetic field up to $8.5 \mathrm{~T}$. This resistance minimum can be well fitted with the Hamann resistivity formula. We suggest that the survival of the magnetic moment of Fe impurities in $\mathrm{Nb}$ is due to their not being fully dissolved into the bcc structure of $\mathrm{Nb}$.

In spite of the strong agreement with the Hamann theory we found in this report, a few words of caution are warranted. First, we do not have direct evidence that the Fe impurity atoms retain their magnetic moments, a direct test (e.g. using spin-polarized STM) would be highly desirable. Second, the formation of the Kondo bound states may be detectable in the change of effective mass of the charge carriers.

\section{Methods}

The five samples used in this paper are from the same $\mathrm{Nb}$ thin film wafer. Our $\mathrm{Nb}$ film has a thickness of $120 \mathrm{~nm}$, deposited on $\mathrm{SiO}_{2} / \mathrm{Si}$ substrates by DC magnetron sputtering, as reported previously ${ }^{77,78}$. The SEM micrographs show that the films are granular in nature, with average grain size around $20 \mathrm{~nm}$ in as-sputtered sample and the grain sizes increase with annealing temperature, up to about $40 \mathrm{~nm}$ at $600{ }^{\circ} \mathrm{C}$, as shown in Supplementary Fig. SI-1 in the Supplementary Information. The $\mathrm{Nb}$ films were patterned into 4-probe devices, using standard photo lithography and ion beam etching. The sample region is a micro-bridge of sizes $1 \mathrm{~mm} \times 10 \mu \mathrm{m}$. The sample was annealed in the thermal annealing chamber filled with flowing argon gas (purity: 99.99\%, pressure: 50 Pa, flow rate: $20 \mathrm{sccm}$ ) at different temperatures $\left(300^{\circ} \mathrm{C}, 400^{\circ} \mathrm{C}, 500{ }^{\circ} \mathrm{C}\right.$ and $\left.600{ }^{\circ} \mathrm{C}\right)$ and for different duration $(30$ $\mathrm{min}$ and $60 \mathrm{~min}$ ). During the annealing, the $\mathrm{Nb}$ film chip was held using an iron clip. Even though the annealing temperature is far below the melting point $\left(1538^{\circ} \mathrm{C}\right)$ for $\mathrm{Fe}$, the bombardment by argon gas molecules was enough to transport a minute amount of Fe into the $\mathrm{Nb}$ film sample. This "co-annealing" method of introducing 
Fe impurities into a host metal is similar to that of a previous experiment ${ }^{79}$. After annealing, the Nb samples were naturally cooled to room temperature under half of an atmosphere pressure of argon gas.

The temperature dependent $(2-100 \mathrm{~K})$ resistances of the samples were measured by standard four-probe method with magnetic field perpendicular to the film surface using a commercial system, the Physical Property Measurement System (PPMS-9, Quantum Design Inc.). The resistances were measured with the AC resistance mode in PPMS. The ohmic resistance measurements were carried out using a current of $120 \mu \mathrm{A}$.

An elemental analysis of the annealed samples using the Time of Flight Secondary Ion Mass Spectrometry (TOF-SIMS) technique was conducted by a commercial service (WinTech Nano, address provided in the caption of Supplementary Fig. SI-3). In this test, $10 \mathrm{~nm}$ of the $\mathrm{Nb}$ film was sputtered away from the surface of annealed sample before taking the TOF-SIMS measurements in order to get the internal information of the sample. For the $\mathrm{Nb}$ film annealed at $600^{\circ} \mathrm{C}$ for $60 \mathrm{~min}$. in Ar atmosphere, the surface density of Fe atoms is about $7-10 \times 10^{-3}$ $\mu \mathrm{m}^{-2}$, see Supplementary Fig. SI-3. This is about $0.1 \mathrm{Fe}$ atom per $1 \mu \mathrm{m} \times 1 \mu \mathrm{m}$ area, or $1 \mathrm{Fe}$ atom per $3.2 \mu \mathrm{m} \times$ $3.2 \mu \mathrm{m}$ area. For the $\mathrm{Nb}$ films annealed at $500{ }^{\circ} \mathrm{C}$ and lower, the TOF-SIMS measurements yielded Fe signals, but too scattered to be reliable, see Supplementary Fig. SI- 4.

Received: 22 April 2021; Accepted: 21 June 2021

Published online: 09 July 2021

\section{References}

1. Balatsky, A. V., Vekhter, I. \& Zhu, J.-X. Impurity-induced states in conventional and unconventional superconductors. Rev. Mod. Phys. 78, 373. https://doi.org/10.1103/RevModPhys.78.373 (2006).

2. Anderson, P. W. Theory of dirty superconductors. J. Phys. Chem. Solids 11, 26-30. https://doi.org/10.1016/0022-3697(59)90036-8 (1959).

3. Matthias, B. T. et al. Magnetic moment of transition metal atoms in dilute solution and their effect on superconducting transition temperature. Phys. Rev. Lett. 5, 542. https://doi.org/10.1103/PhysRevLett.5.542 (1960).

4. Clogston, A. M. et al. Local magnetic moment associated with an iron atom dissolved in various transition metal alloys. Phys. Rev. 125, 541-552. https://doi.org/10.1103/PhysRev.125.541 (1962).

5. Anderson, P. W. Localized magnetic states in metals. Phys. Rev. 124, 41. https://doi.org/10.1103/PhysRev.124.41 (1961).

6. Coles, B. R. Low-temperature resistivity behaviour of molybdenum-iron, niobium-iron and palladium-iron alloys. Philos. Mag. 8, 335-337. https://doi.org/10.1080/14786436308211131 (1962).

7. de Haas, W., de Boer, J. \& \& van dën Berg, G. The electrical resistance of gold, copper and lead at low temperatures. Physica 1, 1115-1124. https://doi.org/10.1016/S0031-8914(34)80310-2 (1934).

8. Kondo, J. Resistance minimum in dilute magnetic alloys. Prog. Theor. Phys. 32, 37-49. https://doi.org/10.1143/PTP.32.37 (1964).

9. Suhl, H. Dispersion theory of the Kondo effect. Phys. Rev. 138, A515-A523. https://doi.org/10.1103/PhysRev.138.A515 (1965).

10. Nagaoka, Y. Self-consistent treatment of Kondo's effect in dilute alloys. Phys. Rev. 138, A1112-A1120. https://doi.org/10.1103/ PhysRev.138.A1112 (1965).

11. Hamann, D. R. New solution for exchange scattering in dilute alloys. Phys. Rev. 158, 570-580. https://doi.org/10.1103/PhysRev. 158.570 (1967).

12. Nozières, P. \& Blandin, A. Kondo effect in real metals. J. Phys. 41, 193-211. https://doi.org/10.1051/jphys:01980004103019300 (1980).

13. Daybell, M. D. \& Steyert, W. A. Observation of Nagaoka's bound state for conduction electrons in dilute magnetic alloys. Phys. Rev. Lett. 18, 398. https://doi.org/10.1103/PhysRevLett.18.398 (1967).

14. Liang, V. \& Tsuei, C. Concentration dependence of Kondo temperature and unitarity limit in non-dilute magnetic amorphous alloys. Solid State Commun. 9, 579-582. https://doi.org/10.1016/0038-1098(71)90149-9 (1971).

15. Liang, V. K. C. \& Tsuei, C. C. Kondo effect in an amorphous $\mathrm{Ni}_{41} \mathrm{Pd}_{41} \mathrm{~B}_{18}$. Phys. Rev. B 7, 3215. https://doi.org/10.1103/PhysRevB.7. 3215 (1973).

16. Sütsch, P. \& Umlauf, E. Transport properties of (La, Pr)Sn 3 alloys at low temperatures. J. Low Temp. Phys. 42, 11-33. https://doi. org/10.1007/BF00116693 (1981).

17. Chandrasekhar, V. et al. Absence of size dependence of the Kondo resistivity. Phys. Rev. Lett. 72, 2053-2056. https://doi.org/10. 1103/PhysRevLett.72.2053 (1994).

18. Chen, J.-H., Li, L., Cullen, W., Williams, E. \& Fuhrer, M. Tunable Kondo effect in graphene with defects. Nat. Phys. 7, 535-538. https://doi.org/10.1038/nphys1962 (2011).

19. Szlawska, M. \& Kaczorowski, D. Antiferromagnetic order and Kondo effect in single-crystalline Ce $\mathrm{IrSi}_{3}$. Phys. Rev. B 84, 094430. https://doi.org/10.1103/PhysRevB.84.094430 (2011).

20. Liang, L. et al. Inducing ferromagnetism and Kondo effect in platinum by paramagnetic ionic gating. Sci. Adv. 4, eaar2030. https:// doi.org/10.1126/sciadv.aar2030 (2018).

21. Yeh, S.-S. et al. Oxygen vacancy-driven orbital multichannel Kondo effect in Dirac nodal line metals $\mathrm{IrO}_{2}$ and $\mathrm{RuO}_{2}$. Nat. Commun. 11, 4749. https://doi.org/10.1038/s41467-020-18407-7 (2020).

22. Ding, X. et al. Crossover from Kondo to Fermi-liquid behavior induced by high magnetic field in $1 T-\mathrm{VTe}_{2}$ single crystals. Phys. Rev. B 103, 125115. https://doi.org/10.1103/PhysRevB.103.125115 (2021).

23. Ren, Z., Taskin, A., Sasaki, S., Segawa, K. \& Ando, Y. Large bulk resistivity and surface quantum oscillations in the topological insulator $\mathrm{Bi}_{2} \mathrm{Te}_{2}$ Se. Phys. Rev. B 82, 241306. https://doi.org/10.1103/PhysRevB.82.241306 (2010).

24. Ren, Z., Taskin, A., Sasaki, S., Segawa, K. \& Ando, Y. Observations of two-dimensional quantum oscillations and ambipolar transport in the topological insulator $\mathrm{Bi}_{2} \mathrm{Se}_{3}$ achieved by Cd doping. Phys. Rev. B 84, 075316. https://doi.org/10.1103/PhysRevB. 84.075316 (2011).

25. Shimamura, K. et al. Electrical control of Curie temperature in cobalt using an ionic liquid film. Appl. Phys. Lett. 100, 075316. https://doi.org/10.1063/1.3695160 (2012).

26. Liu, H., Xue, Y., Shi, J., Guzman, R. \& Gao, H. Observation of the Kondo effect in multilayer single-crystalline VTe 2 nanoplates. Nano Lett. 19, 8572-8580. https://doi.org/10.1021/acs.nanolett.9b03100 (2019).

27. Liu, H. et al. Quasi-2D transport and weak antilocalization effect in few-layered VSe 2 . Nano Lett. 19, 4551-4559. https://doi.org/ 10.1021/acs.nanolett.9b01412 (2019).

28. Cooper, L. N. Bound electron pairs in a degenerate fermi gas. Phys. Rev. 104, 1189-1190. https://doi.org/10.1103/PhysRev.104. 1189 (1956).

29. Bardeen, J., Cooper, L. N. \& Schrieffer, J. R. Microscopic theory of superconductivity. Phys. Rev. 106, 162-164. https://doi.org/10. 1103/PhysRev.106.162 (1957). 
30. Müller-Hartmann, E. \& Zittartz, J. Kondo effect in superconductors. Phys. Rev. Lett. 26, 428-432. https://doi.org/10.1103/PhysR evLett.26.428 (1971).

31. Yazdani, A., Jones, B. A., Lutz, C. P., Crommie, M. F. \& Eigler, D. M. Probing the local effects of magnetic impurities on superconductivity. Science 275, 1767-1770. https://doi.org/10.1126/science.275.5307.1767 (1997).

32. Singh, D. K. \& Tuominen, M. T. Realization of artificial Kondo lattices in nanostructured arrays. Phys. Rev. B 83, 014408. https:// doi.org/10.1103/PhysRevB.83.014408 (2011).

33. Liu, C., Huang, Y., Chen, Y. \& Ting, C. S. Temperature-dependent spectral function of a Kondo impurity in an $s$-wave superconductor. Phys. Rev. B 99, 174502. https://doi.org/10.1103/PhysRevB.99.174502 (2019).

34. Mydeen, K. et al. Electron doping of the iron-arsenide superconductor CeFeAsO controlled by hydrostatic pressure. Phys. Rev. Lett. 125, 207001. https://doi.org/10.1103/PhysRevLett.125.207001 (2020).

35. Farinacci, L. et al. Interfering tunneling paths through magnetic molecules on superconductors: Asymmetries of Kondo and Yu-Shiba-Rusinov resonances. Phys. Rev. Lett. 125, 256805. https://doi.org/10.1103/PhysRevLett.125.256805 (2020).

36. Takayanagi, S., Takano, M., Kimura, Y. \& Sugawara, T. Kondo effect in superconducting dilute MoCo, MoFe, and MoMn alloys. J. Low Temp. Phys. 16, 519-531. https://doi.org/10.1007/BF00654900 (1974).

37. Matsuura, T., Ichinose, S. \& Nagaoka, Y. Theory of Kondo effect in superconductors. I: Transition temperature and upper critical field. Prog. Theor. Phys. 57, 713-733. https://doi.org/10.1143/PTP.57.713 (1977).

38. Bhatia, E., Talapatra, A., Mohanty, J. \& Senapati, K. Superconductivity, Kondo effect, and observation of self-organized pattern formation in intermetallic $\mathrm{NiBi}_{3}$ thin films. Intermetallics 94, 160-164. https://doi.org/10.1016/j.intermet.2018.01.005 (2018).

39. Sun, L. et al. Pressure-induced competition between superconductivity and Kondo effect in $\mathrm{CeFeAsO}_{1-x} \mathrm{~F}_{x}(\mathrm{x}=0.16$ and 0.3$)$.. EPL 91, 57008. https://doi.org/10.1209/0295-5075/91/57008 (2010).

40. Druzhini, A. A., Liakh-Kaguy, N. S., Ostrovskii, I. P., Khoverko, Y. M. \& Rogacki, K. Superconductivity and Kondo effect of Pd $x$ $\mathrm{Bi}_{2} \mathrm{Se}_{3}$ whiskers at low temperatures. JNEP 9, 05013 (2017) https://doi.org/10.21272/jnep.9(5).05013.

41. Osada, M., Wang, B. Y., Lee, K., Li, D. \& Hwang, H. Y. Phase diagram of infinite layer praseodymium nickelate $\operatorname{Pr}_{1-x} \mathrm{Sr}_{x} \mathrm{NiO}_{2}$ thin films. Phys. Rev. Mater. 4, 121801. https://doi.org/10.1103/PhysRevMaterials.4.121801 (2020).

42. Zeng, S. et al. Phase diagram and superconducting dome of infinite-layer $\mathrm{Nd}_{1-x} \mathrm{Sr}_{x} \mathrm{NiO}_{2}$ thin films. Phys. Rev. Lett. 125, 147003. https://doi.org/10.1103/PhysRevLett.125.147003 (2020).

43. Wang, R. et al. Kondo signatures of a quantum magnetic impurity in topological superconductors. Phys. Rev. Lett. 122, 087001. https://doi.org/10.1103/PhysRevLett.122.087001 (2019).

44. Ye, X. et al. Unconventional Josephson junctions with topological Kondo insulator weak links. Phys. Rev. B 100, 104505. https:// doi.org/10.1103/PhysRevB.100.104505 (2019).

45. Cieplak, M. Z., Xiao, G., Bakhshai, A. \& Chien, C. L. Superconducting and normal-state properties of $\mathrm{La}_{1.85} \mathrm{Sr}_{0.15} \mathrm{Cu}_{1-\mathrm{x}} \mathrm{Ga}_{x} \mathrm{BO}_{4}$. Phys. Rev. B 39, 4222-4230 (1989).

46. DeSorbo, W. Effect of dissolved gases on some superconducting properties of niobium. Phys. Rev. 132, 107-121. https://doi.org/ 10.1103/PhysRev.132.107 (1963).

47. Finnemore, D. K., Stromberg, T. F. \& Swenson, C. A. Superconducting properties of high-purity niobium. Phys. Rev. 149, 231-243. https://doi.org/10.1103/PhysRev.149.231 (1966).

48. Webb, G. W. Low-temperature electrical resistivity of pure niobium. Phys. Rev. 181, 1127-1135. https://doi.org/10.1103/PhysRev. 181.1127 (1969).

49. Koch, C. C., Scarbrough, J. O. \& Kroeger, D. M. Effects of interstitial oxygen on the superconductivity of niobium. Phys. Rev. B 9, 888-897. https://doi.org/10.1103/PhysRevB.9.888 (1974).

50. Koch, C. C. \& Kroeger, D. M. The effects of yttrium or gadolinium on the superconducting transition temperature of niobium. J. Less-Common Met. 40, 29-38. https://doi.org/10.1016/0022-5088(75)90179-4 (1975).

51. Karim, D. P., Ketterson, J. B. \& Crabtree, G. W. A de Haas-van Alphen study of niobium: Fermi surface, cyclotron effetive masses, and magnetic breakdown effects. J. Low Temp. Phys. 30, 389-423. https://doi.org/10.1007/BF00114959 (1978).

52. Allen, P. B. \& Dynes, R. C. Transition temperature of strong-coupled superconductors reanalyzed. Phys. Rev. B 12, 905-922. https:// doi.org/10.1103/PhysRevB.12.905 (1975).

53. Jani, A. R., Brener, N. E. \& Callaway, J. Band structure and related properties of bcc niobium. Phys. Rev. B 38, 9425. https://doi. org/10.1103/PhysRevB.38.9425 (1988).

54. Ling, X. S. et al. Nature of phase transitions of superconducting wire networks in a magnetic field. Phys. Rev. Lett. 76, 2989. https:// doi.org/10.1103/PhysRevLett.76.2989 (1996).

55. Santavicca, D. F., Reese, M. O., True, A. B., Schmuttenmaer, C. A. \& Prober, D. E. Antenna-coupled niobium bolometers for Terahertz spectroscopy. IEEE Trans. Appl. Supercond. 17, 412-415. https://doi.org/10.1109/TASC.2007.898191 (2007).

56. Zhu, X. et al. Signature of coexistence of superconductivity and ferromagnetism in two-dimensional $\mathrm{NbSe}_{2}$ triggered by surface molecular adsorption. Nat. Commun. 7, 11210. https://doi.org/10.1038/ncomms11210 (2016).

57. Ziman, J. M. Principles of the Theory of Solids, chapter 7, 2nd Ed. (Cambridge University Press, 1972).

58. Mayadas, A. F. \& Shatzkes, M. Electrical-resistivity model for polycrystalline films: The case of arbitrary reflection at external surfaces. Phys. Rev. B 1, 1382. https://doi.org/10.1103/PhysRevB.1.1382 (1970).

59. Tesanovic, Z., Jaric, M. \& Maekawa, S. Quantum transport and surface scattering. Phys. Rev. Lett. 57, 2760. https://doi.org/10. 1103/PhysRevLett.57.2760 (1986).

60. Kim, Y., Lee, S.-G. \& Choi, J.-H. Ex situ annealing effect on $\mathrm{Nb}$ thin films prepared by DC magnetron sputtering. Phys. C Supercond. Appl. 471, 1193-1195. https://doi.org/10.1016/j.physc.2011.05.156 (2011).

61. Khan, H. et al. Magnetic and superconducting properties of niobium oxides. Mater. Res. Bull. 9, 1129. https://doi.org/10.1016/ 0025-5408(74)90028-2 (1974).

62. Tinkham, M. Introduction to Superconductivity, Chapter 4 (McGraw-Hill, 1996).

63. Honig, J. M., Sinha, A. P. B., Wahnsiedler, W. E. \& Kuwamoto, H. Studies of the band structure of NbO by X-ray photoelectróh spectroscopy. Phys. Status Solidi B 73, 651. https://doi.org/10.1002/pssb.2220730233 (1976).

64. Zhussupbekov, K. et al. Oxidation of $\mathrm{Nb}(110)$ : Atomic structure of the $\mathrm{NbO}$ layer and its influence on further oxidation. Sci. Rep. 10, 3794. https://doi.org/10.1038/s41598-020-60508-2 (2020).

65. Giordano, N. Weak localization and electron-electron interaction effects in thin metal wires and films. Int. J. Mod. Phys. B 24, 2053-2071. https://doi.org/10.1142/S0217979210064691 (2010).

66. McGinnis, W. C. \& Chaikin, P. M. Electron localization and interaction effects in palladium and palladium-gold films. Phys. Rev. B 32, 6319-6330. https://doi.org/10.1103/PhysRevB.32.6319 (1985).

67. Seng, P., Logvenov, G. Y., Tidecks, R., Samwer, K. \& Oboznov, V. Hall effect in superconducting Nb/Cu multilayers. Phys. C 235-240, 2565-2566. https://doi.org/10.1016/0921-4534(94)92503-8 (1994).

68. Wilson, K. The renormalization group: Critical phenomena and the Kondo problem. Rev. Mod. Phys. 47, 773-840. https://doi.org/ 10.1103/RevModPhys.47.773 (1975).

69. Barzykin, V. \& Affleck, I. The Kondo screening cloud: What can we learn from perturbation theory?. Phys. Rev. Lett. 76, 4959. https://doi.org/10.1103/PhysRevLett.76.4959 (1996).

70. Goldhaber-Gordon, D. et al. From the Kondo regime to the mixed-valence regime in a single-electron transistor. Phys. Rev. Lett. 81, 5225-5228. https://doi.org/10.1103/PhysRevLett.81.5225 (1998). 
71. Li, Y. et al. Electrostatic tuning of Kondo effect in a rare-earth-doped wide-band-gap. Phys. Rev. B 87, 155151. https://doi.org/10. 1103/PhysRevB.87.155151 (2013).

72. Han, Y.-L. et al. Carrier-mediated Kondo effect and Hall mobility by electrolyte gating in slightly doped anatase $\mathrm{TiO}_{2}$ films. $P$ hys. Rev. B 90, 205107. https://doi.org/10.1103/PhysRevB.90.205107 (2014).

73. Urkude, R. et al. Observation of Kondo behavior in the single crystals of $\mathrm{Mn}$-doped $\mathrm{Bi}_{2} \mathrm{Se}_{3}$ topological insulator. AIP Adv. 8, 045315. https://doi.org/10.1063/1.5026144 (2018).

74. Nobukane, H., Tabata, Y., Kurosawa, T., Sakabe, D. \& Tanda, S. Coexistence of the Kondo effect and spin glass physics in Fe-doped $\mathrm{NbS}_{2}$. J. Phys. Condens. Matter 32, 165803. https://doi.org/10.1088/1361-648x/ab622a (2020).

75. Barua, S., Hatnean, M. C., Lees, M. R. \& Balakrishnan, G. Signatures of the Kondo effect in VSe 2 . Sci. Rep.https://doi.org/10.1038/ s41598-017-11247-4 (2017).

76. Giordano, N. Magnetoresistance of small Kondo systems. Phys. Rev. B 53, 2487-2491. https://doi.org/10.1103/PhysRevB.53.2487 (1996).

77. He, S. et al. Wire network behavior in superconducting Nb films with diluted triangular arrays of holes. J. Phys. Condens. Matter 24, 155702. https://doi.org/10.1088/0953-8984/24/15/155702 (2012).

78. Zhang, W. et al. Crossover behaviors in magnetoresistance oscillations for $\mathrm{Nb}$ thin film with rectangular arrays of antidots. EPL 99, 37006. https://doi.org/10.1209/0295-5075/99/37006 (2012).

79. Blachly, M. A. \& Giordano, N. Kondo effect in Cu(Fe) films. Phys. Rev. B 49, 6788-6793. https://doi.org/10.1103/PhysRevB.49. 6788 (1994).

\section{Acknowledgements}

We wish to thank Professor Xianggang Qiu of IoP-CAS for providing the as-sputtered Nb films. X.S.L. acknowledges helpful discussions with Prof. Venkat Chandrasekhar and Prof. Ilya Vekhter, and helpful communications with Prof. D. R. Hamann.

\section{Author contributions}

H.S.Z., D.Z., R.J.T., X.S.L. and Z.H.H. designed, discussed and monitored the progress of the research projects. Z.W.H. and Z.X.P. fabricated the Nb thin films. H.S.Z. and D.Z. fabricated Nb film devices. G.Q.L. performed the thermal annealing of the $\mathrm{Nb}$ samples. H.S.Z. performed the transport measurements. H.S.Z. and X.S.L. analyzed the data. H.S.Z., R.J.T. and X.S.L. finalized the manuscript with inputs from all authors.

\section{Competing interests}

The authors declare no competing interests.

\section{Additional information}

Supplementary Information The online version contains supplementary material available at https://doi.org/ 10.1038/s41598-021-93731-6.

Correspondence and requests for materials should be addressed to R.T. or X.S.L.

Reprints and permissions information is available at www.nature.com/reprints.

Publisher's note Springer Nature remains neutral with regard to jurisdictional claims in published maps and institutional affiliations.

(i) Open Access This article is licensed under a Creative Commons Attribution 4.0 International License, which permits use, sharing, adaptation, distribution and reproduction in any medium or format, as long as you give appropriate credit to the original author(s) and the source, provide a link to the Creative Commons licence, and indicate if changes were made. The images or other third party material in this article are included in the article's Creative Commons licence, unless indicated otherwise in a credit line to the material. If material is not included in the article's Creative Commons licence and your intended use is not permitted by statutory regulation or exceeds the permitted use, you will need to obtain permission directly from the copyright holder. To view a copy of this licence, visit http://creativecommons.org/licenses/by/4.0/.

(c) The Author(s) 2021 